\title{
Berlin sieht es locker - Brüssel (noch) nicht
}

Viel wurde bereits darüber diskutiert, wie der Bund zumindest kurzfristig zusätzliche Ausgaben kreditfinanziert tätigen kann, ohne dadurch formal die Vorgaben der Schuldenbremse zu verletzen (Magin, 2010; Boysen-Hogrefe, 2019; SVR, 2021 und viele andere). Mit dem Koalitionsvertrag und dem zweiten Nachtragshaushalt 2021 liegen die Karten auf dem Tisch, wie die Ampelkoalition dies nun anstreben will. Die neue Koalition verlässt sich dabei nicht nur auf ein Vehikel, sondern sucht verschiedene Wege, die von ihr in Aussicht gestellten Projekte per Kredit zu finanzieren.

Zum einen soll die Gelegenheit genutzt werden, dass die Schuldenbremse in der Corona-Zeit ausgesetzt ist und damit die von ihr gesetzten Grenzen für die Nettokreditaufnahme (NKA) überschritten werden können. Damit die finanziellen Spielräume in absehbarer Zeit nicht von den dadurch entstehenden Tilgungsverpflichtungen eingeschränkt werden, gehört es zu den Maßnahmen der neuen Koalition, die Tilgung später zu starten und die Tilgungsfristen deutlich zu verlängern. Allerdings sind die Notlagenkredite auf Bundesebene bisher an das Jahr der Notlage gebunden. Haushaltsrechtlich ist es umstritten, zweckfreie Rücklagen aus der Kreditaufnahme in den Corona-Jahren zu bilden. ${ }^{1}$ Nach den bisherigen Regelungen der Schuldenbremse führte die Befüllung von zweckgebundenen Sondervermögen nicht zu höheren Spielräumen in der Zukunft. Die viel diskutierte Umwidmung von 60 Mrd. Euro durch den zweiten Nachtragshaushalt wäre ohne Wirkung auf die künftigen Spielräume der Ampelkoalition. Aus diesem Grund wird nun die Abrechnung der Schuldenbremse geändert und dies sogar rückwirkend bis ins Jahr 2016, sodass alle seit Beginn der Schuldenbremse initiierten und im Jahr 2021 noch existierenden Sondervermögen des Bundes berücksichtigt werden. Doch was läuft nun anders in der Abrechnung der Schuldenbremse und was hat das für Konsequenzen?

1 Der Staatsgerichtshof des Landes Hessen hat bezüglich der in der Corona-Notsituation zusätzlich aufgenommenen Kredite des Landes Hessen in seinem Urteil vom 27.10.2021 ausgeführt, „dass kreditfinanzierte Maßnahmen zur Krisenbewältigung geeignet, erforderlich und angemessen sein müssten“. Diese Bewertung ist möglicherweise auf den Bund übertragbar und dürfte auf eine allgemeine Rücklage nicht zutreffen.

(c) Der/die Autor:in 2022. Open Access: Dieser Artikel wird unter der Creative Commons Namensnennung 4.0 International Lizenz veröffentlicht (creativecommons.org/licenses/by/4.0/deed.de).

Open Access wird durch die ZBW - Leibniz-Informationszentrum Wirtschaft gefördert.

\section{Corona-Schulden und die Abrechnung der Schuldenbremse}

Die Schuldenbremse des Bundes bezieht sich bislang auf die Nettokreditaufnahme des Kernhaushalts bereinigt um finanzielle Transaktionen (z. B. Erwerb von Wertpapieren) ${ }^{2}$ und konjunkturelle Einflüsse. ${ }^{3}$ Zudem werden die Finanzierungssalden der Sondervermögen des Bundes einbezogen (alt). Letzteres soll nun gestrichen werden, sodass Defizite der Sondervermögen nicht länger die Nettokreditaufnahme im Kernhaushalt schmälern (neu).

Dabei ist Folgendes zu beachten: Die Befüllung der Sondervermögen erfolgt in Form von Kreditermächtigungen. Durch die Befüllung bzw. die neuen Kreditermächtigungen werden die Rücklagen der Sondervermögen erhöht. Die Veränderung des Rücklagenbestands über das Jahr entspricht dem Finanzierungssaldo der Sondervermögen. Die Sondervermögen tätigen also mit der Befüllung nicht in gleichem Umfang sofort Ausgaben, vielmehr bekommen sie die Möglichkeit, im Nennwert der Kreditermächtigungen sofort oder in den Folgejahren Ausgaben zu tätigen, was mit einem entsprechenden Abbau der Rücklagen einhergeht.

Bei der alten Regelung hatte die Befüllung für sich genommen aus Sicht der Schuldenbremse keine Wirkung, da zwar der Kernhaushalt belastet, der Finanzierungssaldo des Sondervermögens aber im gleichen Umfang erhöht wurde. Erst die Ausgaben der Sondervermögen musste sich der Kernhaushalt anrechnen lassen. Letzt-

2 Der von der Ampelkoalition geplante Aufbau eines Kapitalstocks der Rentenversicherung mit $10 \mathrm{Mrd}$. Euro aus Haushaltsmitteln dürfte als finanzielle Transaktion gewertet werden und daher die Spielräume des Kernhaushalts unter der Schuldenbremse nicht einengen.

3 Im Koalitionsvertrag wird eine Überprüfung der derzeit gängigen Methode angeregt. Es gibt keine Vorfestlegung und es wird dabei die Funktionsweise bei systemischen Krisen betont. Ob eine entsprechende Methodenrevision in „normalen“ Zeiten zu mehr Kreditspielräumen führt, ist zweifelhaft.

Prof. Dr. Jens Boysen-Hogrefe ist stellvertretender Direktor des Forschungszentrums Konjunktur und Wachstum am Kiel Institut für Weltwirtschaft und lehrt an der Christian-Albrechts-Universität zu Kiel. 
lich hatte die Befüllung der Sondervermögen eine ähnliche Wirkung wie eine Verpflichtungsermächtigung. Im laufenden Haushalt wird dadurch späteren Haushalten vorgeschrieben, für welchen Bereich diese dann Mittel aufzuwenden haben.

Mit der neuen Regelung muss sich der Kernhaushalt direkt die Befüllung anrechnen lassen, wobei es dann keine Rolle mehr spielt, wann die Sondervermögen in der Folge ihre Rücklagen abbauen. ${ }^{4}$ Die Sondervermögen wirken nun eher wie eine zweckgebundene Rücklage. Bei „normalem" Betrieb der Schuldenbremse, allgemeinem Preisauftrieb und zeitlichen Verzögerungen bei den Ausgaben der Sondervermögen dürfte diese Regelung die Spielräume des Bundes letztlich sogar einengen, da mit der Befüllung der Kernhaushalt zukünftige Ausgaben fix finanziert. Die Preise von morgen müssen schon mit den Steuereinnahmen von heute ausgeglichen werden. Der Effekt der neuen Regelung besteht darin, Kreditermächtigungen aus der Corona-Zeit in die mittlere Frist mitzunehmen, sofern es gelingt, die bestehende Zweckbindung des Sondervermögens mit der Corona-Krise in Verbindung zu bringen.

Dabei geht es nicht nur um 60 Mrd. Euro aus dem Jahr 2021, sondern auch um die Ende 2020 bereits bestehenden Rücklagen der Sondervermögen. Insbesondere 2020 wurde der Energie- und Klimafonds stark aufgefüllt, unter anderem um die Subvention der EEG-Umlage in den Folgejahren abzusichern. Mit der neuen Regelung werden rückwirkend alle Befüllungen der Ende 2020 bestehenden Sondervermögen neu abgerechnet, wodurch sich die NKA von 2016 bis 2020 rückwirkend ändern. Die um 27,7 Mrd. Euro höhere NKA 2020 führt zu höheren Tilgungsanforderungen in gleicher Höhe. Die Änderungen in den Vorjahren schlagen auf das Kontrollkonto der Schuldenbremse durch. Hieraus entstehen keine Tilgungsverpflichtungen.

In der Summe geht es somit bei der Änderung der Abrechnung der Schuldenbremse nicht nur um die Umwidmung von Corona-Schulden des Jahres 2021, sondern auch um Corona-Schulden des Jahres 2020 sowie um einen, wenn auch kleineren Griff in das Kontrollkonto: insgesamt über 90 Mrd. Euro (vgl. Tabelle 1). Zieht man von dieser Summe die Ausgaben der Sondervermögen des Jahres 2021 ab, ergibt sich für die zukünftigen Kreditfinanzierungsspielräume eine Spanne von 70 Mrd. bis 75 Mrd. Euro. Ein Teil dieser Mittel dürfte auch dazu dienen, die Abschaffung der EEG-Umlage zu finanzieren. Dies bedeutet für die Konsument:innen letztlich eine kreditfinanzierte Abgabensenkung.

4 Viele Länder handhaben Sondervermögen entsprechend der neuen Abrechnungsregelung. So haben auch diverse Länder CoronaSondervermögen zur Finanzierung mittelfristiger Defizite aufgebaut (Deutsche Bundesbank, 2021).
Tabelle 1

Einfluss der geänderten Abrechnung der Schuldenbremse

\begin{tabular}{lrrrr} 
& \multicolumn{2}{c}{2020} & \multicolumn{2}{c}{2021} \\
& Alt & Neu & Alt & Neu \\
\hline + NKA Kernhaushalt & 130,5 & 130,5 & 240,2 & 240,2 \\
\hline - Finanzierungssaldo der Sondervermögen & 27,7 & & 60,0 & \\
\hline - Finanzielle Transaktionen & 6,6 & 6,6 & 5,4 & 5,4 \\
\hline - Konjunkturkomponente & 42,6 & 42,6 & 15,7 & 15,7 \\
\hline = Strukturelle NKA & 53,6 & 81,3 & 159,1 & 219,1 \\
\hline - Obergrenze der strukturellen NKA & 11,7 & 11,7 & 12,1 & 12,1 \\
\hline (0,35\% des BIP) & 41,9 & 69,6 & 147 & 207 \\
\hline = Überschreitung der zulässigen NKA & & 27,7 & & 60,0 \\
\hline Differenzen Neu zu Alt & & & & 87,7 \\
\hline Summe der Differenzen 2020 und 2021 & & & & 4,3 \\
\hline Summe der Differenzen 2016 bis 2019 & & & & 92,0 \\
\hline Gesamtdifferenz & & & &
\end{tabular}

Quelle: Januar- und Septemberausgaben des Monatsberichts des Bundesfinanzministeriums 2017 bis 2021; eigene Berechnungen.

\section{Auftragsverschuldung}

Die Kreditermächtigungen für die Sondervermögen stammen aus dem Bundeshaushalt und werden daher letztlich von der Schuldenbremse erfasst. Es ist allerdings auch möglich, Kredite auf staatliches Geheiß aufzunehmen, die an der Schuldenbremse vorbeilaufen, sofern dies Unternehmen tun, die in staatlichem Besitz oder Zweckgesellschaften sind, die eine eigene Kreditermächtigung haben. Zweckgesellschaften, deren einziger Zweck die Kreditbeschaffung ist, sind rechtlich wohl nicht möglich (Kube, 2021), doch hat die Ampelkoalition bereits klare Projekte definiert, die über öffentliche Unternehmen finanziert werden sollen. Letztlich ist dies nicht neu. Die im Koalitionsvertrag genannten KfW und Deutsche Bahn AG (DB) sind Unternehmen im Besitz des Bundes, die sich bereits fremd finanzieren können und dies auch tun. Ihre Rolle für Klimaschutz und Verkehrswende soll erweitert werden und damit wohl auch deren Verschuldung. Hinzu tritt die Bundesanstalt für Immobilienaufgaben (BImA), die - teilweise kreditfinanziert - staatlich gelenkt Wohnungsbau betreiben soll. Die BImA wird dazu mit einer Verschuldungsmöglichkeit ausgestattet, die somit nicht von der Schuldenbremse erfasst wird.

Eigene Schulden bedeuten auch eigenes Risiko. Zumindest bei den privaten Unternehmen im Staatsbesitz ist eine Haftung des Bundes für Fremdkapital nicht vorgesehen. Typischerweise sind die Finanzierungsbedingungen 
öffentlicher Unternehmen schlechter als die des Bundes. Allerdings kann der Bund Bürgschaften geben, die zunächst weder die NKA noch den Schuldenstand des Bundes tangieren, oder öffentliche Unternehmen mit zusätzlichem Eigenkapital ausstatten, was dann im Sinne der Schuldenbremse als finanzielle Transaktion gesehen wird. So hat die Vorgängerregierung 2021 der DB Eigenkapitalhilfen zukommen lassen, die nicht der Schuldenbremse unterlagen.

Eine weitere Möglichkeit für zusätzliche Verschuldung besteht durch Öffentlich-Private-Partnerschaften (ÖPP). Es sind private Gesellschaften, die nur für den Zweck zur Abwicklung eines staatlichen Auftrags bestehen. Diese Gesellschaften können sich selbständig am Kapitalmarkt verschulden und bekommen Finanzzusagen des Staates. Bei Autobahnprojekten, wie dem Ausbau der A1, wurden z.B. die Einnahmen aus der Lkw-Maut aus den Jahren nach Fertigstellung des Bauprojekts den privaten Gesellschaften zugesprochen. Der Staat verschuldet sich somit effektiv bei den ÖPP. Die Wirtschaftlichkeit von ÖPP wird immer wieder angezweifelt, auch weil die Kreditkosten zumindest seit der Finanzkrise 2008/2009 für Private deutlich höher sind als für den Bund. Der Koalitionsvertrag äußert sich eher vorsichtig zu dieser Möglichkeit, schließt sie aber nicht aus.

Wie wird das in Brüssel gesehen?

Bei den bestehenden Fiskalregeln auf europäischer Ebene gilt ein besonderes Augenmerk dem Finanzierungssaldo des Gesamtstaats in Abgrenzung der Volkswirtschaftlichen Gesamtrechnungen (VGR). Sofern der strukturelle Finanzierungssaldo von den Vorgaben (bei mäßigem Bruttoschuldenstand gilt eine als Mittelfristziel bezeichnete Obergrenze von 0,5\% in Relation zum Bruttoinlandsprodukt) abweicht, soll der Finanzierungssaldo in Schritten von bis zu über 1 Prozentpunkt dahin zurückgeführt werden. Im Jahr 2022 ist die fiskalische Überwachung noch ausgesetzt, doch ab 2023 sollen die öffentlichen Haushalte nach jetzigem Stand wieder an den Mittelfristzielen orientiert werden.

Das ist brisant, weil im Rechenwerk der VGR viele Gestaltungsmöglichkeiten der Schuldenbremse nicht zum Zuge kommen. Statt um den Zeitpunkt von Haushaltsentscheidungen geht es in den VGR um den Zeitpunkt der Mittelverwendung (Accrual Principle). Somit treten die Finanzierungsdefizite nicht bei der Befüllung von Sondervermögen auf, sondern wenn die Mittel verausgabt werden. Es spielt somit für die Haushaltsüberwachung keine Rolle, ob Kreditermächtigungen aus Corona-Zeiten in die mittlere Frist mitgenommen werden oder nicht. Es zählt nur der Finanzierungssaldo des jeweils laufenden Jahres. Angesichts zusätzlicher, mittelfristiger Ausgaben via Sondervermögen des Bundes, bereits bestehender Rücklagen bei Bund und Ländern und in der Rentenversicherung, die in den kommenden Jahren abgebaut werden dürften, ist es unwahrscheinlich, dass das mittelfristige Budgetziel 2023 erreicht wird bzw. hinreichend große Konsolidierungsschritte erfolgen (Unabhängiger Beirat des Stabilitätsrats, 2021; Ademmer et al., 2021). Eine kuriose Möglichkeit bestünde darin, das Defizit im letzten Jahr ohne Überwachung, also 2022, kräftig auszuweiten, sodass dann in den Folgejahren größere Schritte leichter fallen.

Die Vorgaben aus Brüssel sind noch mit weiteren Verschuldungsmöglichkeiten der Schuldenbremse über Kreuz. So werden ÖPP typischerweise dem Sektor Staat in den VGR zugerechnet, und somit auch deren Finanzierungssalden. Nur Unternehmen in Staatshand oder Staatsauftrag, die hinreichend viele geschäftliche Verbindungen zum Privatsektor haben, zählen in den VGR nicht zum Staat (Schmidt et al., 2017), so z.B. die KfW und die DB. Sobald die betroffenen Unternehmen mit privaten Unternehmen in Konkurrenz treten können, unterliegen sie der europäischen Wettbewerbsaufsicht. Zuwendungen des Staats, z.B. in Form von Bürgschaften oder Eigenkapitalhilfen sind in diesem Fall deutlich erschwert. So haben die Wettbewerbshüter:innen der EU-Kommission jüngst die Eigenkapitalhilfen für die DB zurechtgestutzt. Zudem grenzt Eurostat in den VGR finanzielle Transaktionen teilweise anders ab. Die DB-Eigenkapitalhilfen wurden nicht als Erwerb von Finanzvermögen gezählt und belasteten somit den staatlichen Finanzierungssaldo.

Das bestehende Regelwerk der Europäischen Union steht somit vielen der Möglichkeiten entgegen, die sich die Ampelkoalition im Hinblick auf die Schuldenbremse geschaffen hat bzw. schaffen will. Die im Koalitionsvertrag in Aussicht gestellte Weiterentwicklung der fiskalpolitischen Regeln auf europäischer Ebene ist vermutlich auch vor diesem Hintergrund zu verstehen.

\section{Fazit}

Für 2022 dürfte es keine bedeutenden Unterschiede machen, ob und wie der Klima- und Transformationsfonds (KTF) als Nachfolger des Energie- und Klimafonds (EKF) befüllt wurde oder wie die Verschuldung der BImA zu werten ist. Denn die abermalige Aussetzung der Schuldenbremse und der fiskalischen Vorgaben auf europäischer Ebene ermöglicht die Defizitfinanzierung über den Kernhaushalt. Teilweise gab es ein solches Vorgehen mit dem Zukunftspaket auch bereits in den Zeiten der Großen Koalition (Boysen-Hogrefe, 2021). Der Unterschied zu der bisherigen Finanzpolitik liegt vielmehr in der Schaffung von bzw. dem stärkeren Rückgriff auf Möglichkeiten, über 
das Jahr 2022 hinaus Projekte per Kredit zu finanzieren, wenn der Kernhaushalt bereits die Grenzen der Schuldenbremse in „normalen“ Zeiten erreicht hat. Ob der Rückgriff auf diese Möglichkeiten ökonomisch klug ist, wird stark vom künftigen Auslastungsgrad der Wirtschaft abhängen. Dabei ist noch nicht ausgemacht, in welchem Umfang die Kreditmöglichkeiten tatsächlich genutzt werden. Daher ist ungewiss, ob es zu einem Konflikt zu den bestehenden europäischen Fiskalregeln kommen wird. Dass diese Möglichkeiten geschaffen werden, macht es aber wahrscheinlicher, dass die Bundesregierung eine Lockerung der europäischen Fiskalregeln anstreben wird - schon aus dem Eigeninteresse, einen Konflikt mit den europäischen Regeln zu vermeiden.

\section{Literatur}

Ademmer, M. et al. (2021), Deutsche Wirtschaft im Winter 2021: Erholung vorerst ausgebremst, Kieler Konjunkturbericht, 86.
Boysen-Hogrefe, J. (2019), Schuldenbremse und "goldene Regel" müssen kein Widerspruch sein, Blogbeitrag, Makronom.

Boysen-Hogrefe, J. (2021), Bundeshaushalt 2021: Zwischen Krisenbewältigung und günstiger Gelegenheit, Kurzgutachten für die Initiative Neue Sozial Marktwirtschaft, IfW Kiel.

Deutsche Bundesbank (2021), Länderfinanzen 2020: Defizit aufgrund temporärer Pandemieeinflüsse, Ausnahmeklauseln auch für Reservebildung genutzt, in Monatsbericht Oktober 2021, 15-42.

Kube, H. (2021), Die Zukunft der staatlichen Kreditaufnahme: Optionen, Grenzen, Kritik, https://verfassungsblog.de/die-zukunft-der-staatlichen-kreditaufnahme/ (23. Dezember 2021).

Magin, C. (2010), Die Wirkungslosigkeit der neuen Schuldenbremse Warum die Staatsverschuldung weiterhin ungebremst steigen kann, Wirtschaftsdienst, 90(4), 262-268, https://www.wirtschaftsdienst.eu/ inhalt/jahr/2010/heft/4/beitrag/die-wirkungslosigkeit-der-neuenschuldenbremse.html (23. Dezember 2021).

Schmidt, P., N. Heil, D. Schmidt und J. Kaiser (2017), Die Abgrenzung des Staatskontos in den Volkswirtschaftlichen Gesamtrechnungen - Zuordnungskriterien für öffentliche Einheiten, Wirtschaft und Statistik, 1, 35-48.

SVR - Sachverständigenrat zur Begutachtung der gesamtwirtschaftlichen Entwicklung (2021), Jahresgutachten 2021/2022: Transformation gestalten: Bildung, Digitalisierung und Nachhaltigkeit, Kapitel 2.

Unabhängiger Beirat des Stabilitätsrats (2021), 17. Stellungnahme zur Einhaltung der Obergrenze für das strukturelle gesamtstaatliche Finanzierungsdefizit.

Title: Berlin Takes It Easy - Brussels (Still) Does Not

Abstract: Various ways exist to make use of public debt financing despite the existence of the debt brake. These are outlined in the recent coalition agreement, which indicates that the new government will go in this direction. Particularly, the accounting of federal special funds will change and the role of public enterprises will increase. Budget surveillance at the European level, however, uses alternative accounting that remains unaffected. German budgets will likely run up against the current European rules in the medium run. Thus, the new budget plans also hint at the readiness to renegotiate fiscal rules for the European Union. 